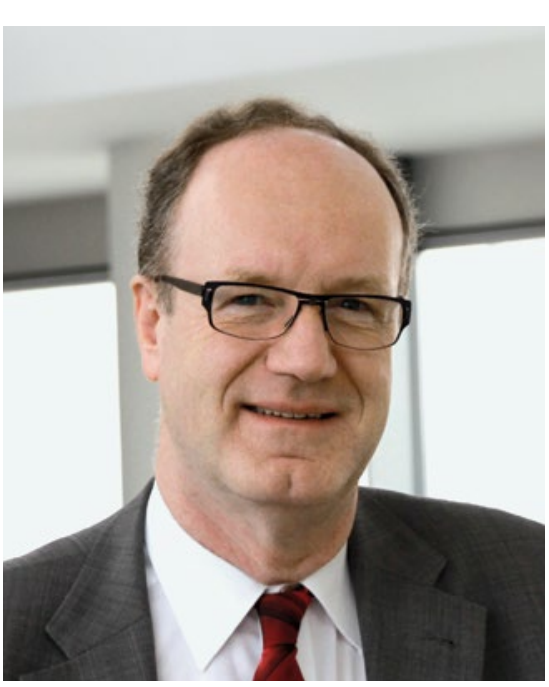

Hans-Georg Frischkorn ist Strategie- und Veränderungsberater in Frankfurt am Main.

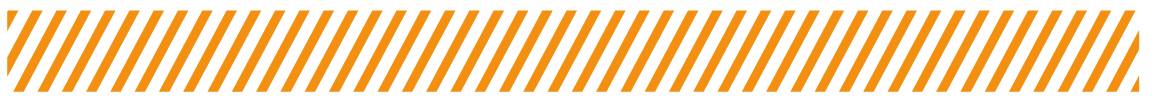

\title{
„Nicht nur eine Herausforderung, sondern vielmehr eine klare Notwendigkeit“
}

ATZelektronik_Herr Frischkorn, die meisten Vorträge und Artikel beginnen seit Jahren mit erschöpfenden Ausführungen zur enorm steigenden Komplexität künftiger Entwicklungen. Sie ergänzen: Ohne konsequent angewandtes Systems Engineering bekommt die Branche die entsprechenden Herausforderungen gar nicht in den Griff. FRISCHKORN_Es ist nicht nur eine Herausforderung, sondern mehr noch eine klare Notwendigkeit, der sich die Automobilindustrie unbedingt stellen muss. Ich weiß, wie schwer es ist, dem alläglichen Umsetzungsdruck auf die Funktionsentwicklungen nachzukommen. Zudem möchte jeder schnell sein. Da bleibt kaum Zeit für die grundlegende Erarbeitung besserer Methoden. Die Unternehmen glauben, nur das eine oder das andere tun zu können.

Dies klingt nicht hoffnungsvoll. Fällt das den Autoherstellern irgendwann mal auf die Füße? Wenn die Autohersteller und ausnahmslos alle Beteiligten das Systems Engineering nicht konsequent anwenden, lassen sich die Träume des hochautomatisierten und hochvernetzten Fahrens einfach nicht realisieren. Das gilt für das Anforderungsmanagement und die Entwicklungsmethoden ebenso wie für die Methoden beim Testen und Validieren. Die große Herausforderung besteht auch darin, gemeinsam an Prozessen zu arbeiten. Nur dann kann die dringend erforderliche Standardisierung von Bausteinen gelingen. Die Komplexität ist schon hoch genug. Wenn die Zulieferer sich dann noch auf jeden OEM und seine Prozesse einstellen müssen, wird das Ganze nicht mehr überschaubar.

Derartige Aufrufe haben wir vor zehn Jahren schon publiziert.

Mit dem Unterschied, dass es heute noch ernster geworden ist. Und der Druck auf dem Kessel sehr hoch ist.

Wie kann man die Veränderungen jetzt noch forcieren? Eine Task Force gründen? Es ist nun wichtig, dass ein gemeinsames Bewusstsein für die Tragweite der Problematik entsteht. Dafür sind Publikationen wie diese und andere in Fach- zeitschriften immer wieder hilfreich. Ebenso Tagungen wie zuletzt die ATZFahrerassistenztagung, wo beispielsweise pragmatisch über das Schlüsselthema Testen in diesem Zusammenhang gesprochen wurde. Offenheit ist wichtig. Ebenso gilt es, immer wieder zu Diskussionen zu motivieren. Derzeit entsteht ein neuer Dialog. Ich kenne zudem vielversprechende Forschungsprojekte, die sich mit Systems Engineering für „system of systems“ beschäftigen.

Jeder möchte schnell sein, sagen Sie. Die Roadmap zum hochautomatisierten Fahren ist beispielsweise zu optimistisch gerechnet, was zu hohe Erwartungen weckt.

Schnelligkeit der Umsetzung ist genauso wichtig wie absolute Qualität und Zuverlässigkeit. Diesen Anspruch erfüllen die in der Automobilindustrie eingesetzten Methoden nur teilweise.

... weil es auch schwierige Passagen gibt? Unter anderem: Schwierig ist die Gesamtfunktionalität eines Fahrzeugs formal und die kritischen Teile eines Fahrzeugs hochformal zu spezifizieren, und diese gleichzeitig mit einer weniger formalen Spezifikation von weniger kritischen Komponenten zu verknüpfen.

Was muss das Topmanagement tun? Das Topmanagement muss sich in dem erwähnten Dialog aktiv einbringen. Das ist unter anderem auch deswegen wichtig, weil mittlerweile branchenfremde und strategisch außerordentlich wichtige Unternehmen in die Prozesskette integriert sind - Internet- und IT-Firmen, Unternehmen der Telekommunikationsund Consumer-Industrie. Hier sehe ich dringenden Handlungsbedarf. Denn diese Firmen müssen die heutigen hohen automobilspezifischen Anforderungen und Methoden kennen. Eine Kette ist nur so stark wie ihr schwächstes Glied.

Herr Frischkorn, ich bedanke mich für das Gespräch.

INTERVIEW: Markus Schöttle FOTO: ESG 